T. Asai

Nagoya Math. J.

Vol. 71 (1978), 149-167

\title{
ON THE DOI-NAGANUMA LIFTING ASSOCIATED WITH IMAGINARY QUADRATIC FIELDS
}

\author{
TETSUYA ASAI
}

\section{Introduction}

Similarly to the real quadratic field case by Doi and Naganuma ([3], [9]) there is a lifting from an elliptic modular form to an automorphic form on $S L_{2}(C)$ with respect to an arithmetic discrete subgroup relative to an imaginary quadratic field. This fact is contained in his general theory of Jacquet ([6]) as a special case. In this paper, we try to reproduce this lifting in its concrete form by using the theta function method developed first by Niwa ([10]); also Kudla ([7]) has treated the real quadratic field case on the same line. The theta function method will naturally lead to a theory of lifting to an orthogonal group of general signature (cf. Oda [11]), and the present note will give a prototype of non-holomorphic case.

Let an imaginary quadratic number field be fixed once for all throughout this paper, and let $\mathfrak{o}$ denote the ring of integers of the field. For simplicity's sake we assume that the class number is one and the discriminant $-D$ is odd prime, less than -3 . For a positive even integer $\nu$ we denote by $\mathscr{S}_{\nu+1}$ the space of cusp forms of weight $\nu+1$ of Neben type $\chi$ with respect to $\Gamma_{0}(D)$, where $\chi$ denotes the Kronecker character $\left(\frac{-D}{*}\right)$. We shall show that each cusp form $f$ in $\mathscr{S}_{\nu+1}$ can be lifted to a $C^{2 \nu+1}$-valued automorphic form $F$ on $S L_{2}(C)$ with respect to $S L_{2}(\mathfrak{o})$, belonging to an irreducible representation of $S U_{2}(C)$ of degree $2 \nu+1$, which is also an eigen-function of the Casimir operator with the eigen-value $\frac{1}{2}\left(\nu^{2}-1\right)$. We shall give the Fourier expansion of $F$ explicitly. It may be remarkable that the lifted image $F$ is cuspidal if and only if $f$ is orthogonal to $\theta_{-D}^{(\nu)}$ in $\mathscr{S}_{\nu+1}$, where $\theta_{-D}^{(\nu)}(z)=\frac{1}{2} \sum_{r \in 0} r^{\nu} \exp (2 \pi i r \bar{r} z)$.

In our argument, a special polynomial of four variables, which is

Received August 2, 1977. 
nothing but a classical Laplace's spherical harmonics in essential, plays a fundamental role, so that we shall devote the first section to summarizing its properties. One of virtues of Niwa's method is in connecting a theta function with so-called Rankin's method, and we shall follow this, but in a little more direct fashion, namely we try to avoid using Eisenstein series there. The exceptional behaviour of $\theta_{-D}^{(\nu)}$ is related to the fact that $\theta_{-D}^{(\nu)}$ is the unique primitive form in $\mathscr{S}_{\nu+1}$ whose Fourier coefficients are all real. As an application of the Doi-Naganuma lifting we shall give a proof of this in the last section.

\section{§1. The spherical harmonic polynomial}

1.1. We shall denote by $\rho_{n}(g)$ the $n$-ply symmetric tensor product of $g \in G L_{2}(C)$, i.e.

$$
{ }^{t}\left((a, b)^{t} g\right)_{n}=\rho_{n}(g)^{t}(a, b)_{n}
$$

with indeterminants $a, b \in C$ and $(a, b)_{n}=\left(a^{n}, a^{n-1} b, \cdots, a b^{n-1}, b^{n}\right)$. Put $G=S L_{2}(C)$ and $K=S U_{2}(C)$. Each $\rho_{n}$ gives an irreducible representation of $K$ as is well known. Let us put

$$
V=\left\{X \in M_{2}(C) ; X={ }^{t} \bar{X}\right\},
$$

which is a vector space of dimension four over $\boldsymbol{R}$, and so we regard $M_{2}(C)$ as $V_{c}=V \otimes_{R} C$. The group $G$ acts on $V$ in such a way that $X^{g}={ }^{t} \bar{g} X g$ for $X \in V$ and $g \in G$. The action restricted to $K$ gives a representation on $V$ equivalent to $\rho_{0} \oplus \rho_{2}$. Let us define two symmetric bilinear forms $Q$ and $R$ on $V$ by

$$
Q(X, Y)=-\operatorname{tr}(X \tilde{Y}), R(X, Y)=\operatorname{tr}(X Y) ; \quad X, Y \in V,
$$

where $\tilde{Y}=\left({ }_{1}{ }^{-1}\right)^{t} Y\left({ }_{-1}{ }^{1}\right) . \quad Q(X)$ and $R(X)$ denote the associated quadratic forms $Q(X, X)$ and $R(X, X)$, respectively. The form $Q$ is of signature $(3,1)$ and $G$-invariant, i.e. $Q\left(X^{g}\right)=Q(X)$ for every $g \in G$, while the form $R$ is positive definite and $K$-invariant, i.e. $R\left(X^{\star}\right)=R(X)$ for every $\kappa \in K$. We should note the form $R\left(X^{g}\right)$ is a minimal majorant of $Q(X)$ for each $g \in G$. For a non-negative integer $\nu$, let $\mathscr{H}^{\nu}$ denote the $C$-linear space of polynomial functions on $V$ spanned by $Q(X, A)^{\nu}$, where $A \in V_{c}$ such that $Q(X, A)=R(X, A)$ and $Q(A)=R(A)=0$. An element of $\mathscr{H}^{\nu}$ is called a spherical harmonic polynomial with respect to $Q$ and its majorant $R$. The dimension of $\mathscr{H}^{\nu}$ is $2 \nu+1$, in fact, we can get a natural basis 
as follows.

Let us put $A={ }^{t}(a, b)(a, b)\left(_{1}{ }^{-1}\right) \in V_{c}$, then the form $Q(X, A)^{\nu}$ is a homogeneous polynomial of degree $\nu$ (resp. $2 \nu$ ) with respect to $X$ (resp. $a, b)$. Hence we may define a homogeneous polynomial $\eta_{\nu, \alpha}(X)$ of degree $\nu$ as the coefficient of $a^{\nu-\alpha} b^{\nu+\alpha}$ in $Q(X, A)^{\nu}$ for each $\alpha,|\alpha| \leqq \nu$. We thus put:

$$
\begin{aligned}
& \eta_{(\nu)}(X)^{t}(a, b)_{2 \nu}=Q(X, A)^{\nu}, \\
& \eta_{(\nu)}(X)=\left(\eta_{\nu,-\nu}(X), \cdots, \eta_{\nu, \nu}(X)\right) .
\end{aligned}
$$

Obviously we have

LEMMA 1. The polynomials $\eta_{\nu, \alpha}(X),|\alpha| \leqq \nu$, form a basis of $\mathscr{H}^{\nu}$.

Since the forms $Q$ and $R$ are $K$-invariant, $\mathscr{H}^{\nu}$ is regarded as a representation space of $K$, which is irreducible. In particular, we have

LEMMA 2. $\eta_{(\nu)}\left(X^{\kappa}\right)=\eta_{(\nu)}(X) \rho_{2 \nu}(\kappa)$ for every $\kappa \in K$.

Proof. We have $\eta_{(\nu)}\left(X^{*}\right)^{t}(a, b)_{2 \nu}=Q\left(X^{s}, A\right)^{\nu}=Q\left(X, A^{\varepsilon^{-1}}\right)^{\nu}$, which is equal to $\eta_{(\nu)}(X)^{t}\left((a, b)^{t} \kappa\right)_{2 \nu}=\eta_{(\nu)}(X) \rho_{2 \nu}(\kappa)^{t}(a, b)_{2 \nu}$, since $A^{\varepsilon^{-1}}={ }^{t}\left((a, b)^{t} \kappa\right)\left((a, b)^{t} \kappa\right)$ $\cdot\left({ }_{1}^{-1}\right)$ for $\kappa \in K$.

We should note that Lemmas 1 and 2 characterize the polynomials $\eta_{\nu, \alpha}(X)$ up to a constant multiple, in fact, by a simple observation we can show that the polynomials $\eta_{\nu, \alpha}(X)$ are essentially the same as classical Laplace's spherical harmonics (cf. [4], Chap. XI).

1.2. We write a general element $X$ of $V$ as follows:

$$
X=\left(\begin{array}{ll}
m & r \\
\bar{r} & n
\end{array}\right) ; m, n \in \boldsymbol{R}, r \in \boldsymbol{C},
$$

and this parametrization will be kept throughout the paper. It is, then, easy to see that $\eta_{(0)}(X)=1$ and $\eta_{(1)}(X)=(-\bar{r}, m-n, r)$, while we can compute more by use of a recursion formula

$$
\eta_{\nu, \alpha}(X)=(-\bar{r}) \eta_{\nu-1, \alpha+1}(X)+(m-n) \eta_{\nu-1, \alpha}(X)+(r) \eta_{\nu-1, \alpha-1}(X)
$$

where we understand $\eta_{0,0}(X)=1$ and $\eta_{\nu, \alpha}(X)=0$ if $|\alpha|>\nu$. The following is an explicit formula which we need later.

LEMMA 3. For $|\alpha| \leqq \nu$, we have 


$$
\eta_{\nu, \alpha}(X)=\nu ! \sum_{\beta, \gamma} \frac{1}{(\alpha+\beta) ! \cdot r !} 2^{-\beta} r^{\alpha} L_{\beta}^{(\alpha)}(2 r \bar{r}) H_{r}(m-n),
$$

where $L_{\beta}^{(\alpha)}$ and $H_{r}$ are Laguerre's and Hermite's polynomials respectively, and the sum is taken over all non-negative integers $\beta, \gamma$ such that $2 \beta$ $+\gamma=\nu-\alpha$ and $\alpha+\beta \geqq 0$.

Proof. We put $q=m-n$ for abbreviation. By the definition (1) we have

$$
\exp \left(\left(-\bar{r} a^{2}+q a b+r b^{2}\right) t\right)=\sum_{\nu=0}^{\infty} \sum_{\alpha=-\nu}^{\nu} \eta_{\nu, \alpha}(X) a^{\nu-\alpha} b^{\nu+\alpha} \frac{t^{\nu}}{\nu !} .
$$

It follows from a generating function formula of $H_{r}$ (e.g. [8], p. 253) that

$$
\exp \left(q a b t-\frac{1}{2} a^{2} b^{2} t^{2}\right)=\sum_{r=0}^{\infty} H_{r}(q) a^{r} b^{r} \frac{t^{r}}{r !} .
$$

On the other hand, we have

$$
\begin{aligned}
\exp \left(\left(-\bar{r} a^{2}+r b^{2}\right) t+\frac{1}{2} a^{2} b^{2} t^{2}\right) & =\sum_{\ell=0}^{\infty} \exp \left(-\bar{r} a^{2} t\right)\left(1+\frac{1}{2} r^{-1} a^{2} t\right)^{\ell} \frac{\left(r b^{2} t\right)^{\ell}}{\ell !} \\
& =\sum_{\ell=0}^{\infty} \sum_{\beta=0}^{\ell} 2^{-\beta} r^{\ell-2 \beta} L_{\beta}^{(\ell-2 \beta)}(2 r \bar{r}) \alpha^{2 \beta} b^{2 \ell-2 \beta} \frac{t^{\ell}}{(\ell-\beta) !}
\end{aligned}
$$

by use of a generating function formula of $L_{\beta}^{(\alpha)}$ (e.g. [8], p. 242). Equating these, we obtain the proof.

For convenience' sake let us define another quadratic form on $V$ by

$$
P(X)=Q(X)+R(X),
$$

then it can be immediately seen that $P$ is $K$-invariant, and $0 \leqq P(X)$ $\leqq 2 R(X)$ for $X \in V$.

LEMMA 4. $\left|\eta_{\nu, \alpha}(X)\right|^{2} \leqq\left(\begin{array}{c}2 \nu \\ \nu-\alpha\end{array}\right)\left(\begin{array}{c}2 \nu \\ \nu\end{array}\right)^{-1} P(X)^{\nu}$ for $X \in V$.

Proof. It is sufficient to prove

$$
\sum_{\alpha=-\nu}^{\nu}\left(\begin{array}{c}
2 \nu \\
\nu-\alpha
\end{array}\right)^{-1}\left|\eta_{\nu, \alpha}(X)\right|^{2}=\left(\begin{array}{c}
2 \nu \\
\nu
\end{array}\right)^{-1} P(X)^{\nu}
$$

Denote by $J$ a diagonal constant matrix of degree $2 \nu+1$ satisfying $(a, b)_{2 v} J^{2 t}(\bar{a}, \bar{b})_{2 \nu}=(a \bar{a}+b \bar{b})^{2 \nu}$, then $J \rho_{2 \nu}(\kappa) J^{-1}$ is a unitary matrix for every 
$\kappa \in K$. Hence the left-hand side of (6), which is $\eta_{(\nu)}(X) J^{-2} \overline{{ }^{t} \eta_{(\nu)}(X)}$, is $K$ invariant as well as the right-hand side. We may, therefore, assume $X=\left(\begin{array}{cc}m & 0 \\ 0 & n\end{array}\right)$. Then both sides are equal to $\left(\begin{array}{c}2 \nu \\ \nu\end{array}\right)^{-1}(m-n)^{2 \nu}$. This completes the proof.

1.3. For the purpose to give another property of $\eta_{\nu, \alpha}(X)$ we recall some notions on Lie derivatives. Let $S$ be an arbitrary element of the Lie algebra of $G$, i.e. $S \in M_{2}(C)$ with $\operatorname{tr}(S)=0$. A differential operator $S^{\prime}\left(\right.$ or $\left.S^{\prime \prime}\right)$ on $C^{\infty}(G)$ is defined by $S^{\prime} f(g)=\left[\frac{\partial}{\partial t} f(g \cdot \exp (t S))\right]_{t=0}($ or by replacing $\frac{\partial}{\partial t}$ with $\frac{\partial}{\partial \bar{t}}$, accordingly.) On the other hand, a function $F \in C^{\infty}(V)$ induces a function $F_{X}(g)=F\left(X^{g}\right) \in C^{\infty}(G)$ for each $X \in V$. Hence $S^{\prime}$ (or $S^{\prime \prime}$ ) operates on $C^{\infty}(V)$ in such way that $\left(S^{\prime} F\right)_{X}=S^{\prime} F_{X}$ (or $\left(S^{\prime \prime} F\right)_{X}=S^{\prime \prime} F_{X}$ ). It can be easily seen that $S^{\prime}$ (or $S^{\prime \prime}$ ) on $C^{\infty}(V)$ is equal to $m^{\prime} \frac{\partial}{\partial m}+n^{\prime} \frac{\partial}{\partial n}+r^{\prime} \frac{\partial}{\partial r}+r^{\prime \prime} \frac{\partial}{\partial \bar{r}}$, where $X S$ (or $\left.{ }^{t} \bar{S} X\right)=\left(\begin{array}{ll}m^{\prime} & r^{\prime} \\ r^{\prime \prime} & n^{\prime}\end{array}\right)$ with the parametrization $X=\left(\begin{array}{cc}m & r \\ \bar{r} & n\end{array}\right) \in V . \quad$ The elements $A=\left(\begin{array}{ll}0 & 1 \\ 0 & 0\end{array}\right), B=\left(\begin{array}{ll}0 & 0 \\ 1 & 0\end{array}\right)$ and $U=\left(\begin{array}{rr}1 & 0 \\ 0 & -1\end{array}\right)$ form a basis of the Lie algebra, and the Casimir operators $C^{\prime}$ and $C^{\prime \prime}$ are given by $C^{\prime}=A^{\prime} B^{\prime}+B^{\prime} A^{\prime}+\frac{1}{2} U^{\prime} U^{\prime}$ and $C^{\prime \prime}=A^{\prime \prime} B^{\prime \prime}$ $+B^{\prime \prime} A^{\prime \prime}+\frac{1}{2} U^{\prime \prime} U^{\prime \prime}$, while both the operators $C^{\prime}$ and $C^{\prime \prime}$ coincide with each other on $C^{\infty}(V)$. Further, by an easy computation we can obtain a formula on $C^{\infty}(V)$ :

$$
C^{\prime}=C^{\prime \prime}=\frac{1}{2} L^{2}+L-\frac{1}{2} Q(X) \Delta_{Q},
$$

where $L=m \frac{\partial}{\partial m}+n \frac{\partial}{\partial n}+r \frac{\partial}{\partial r}+\bar{r} \frac{\partial}{\partial \bar{r}}$ and $\Delta_{Q}=2 \frac{\partial^{2}}{\partial r \partial \bar{r}}-2 \frac{\partial^{2}}{\partial m \partial n}$ (cf. [13], p. 95).

\section{LEMMA 5. Put}

$$
\eta_{\nu, \alpha}^{*}(X)=\eta_{\nu, \alpha}(X) P(X)^{-\left(\nu+\frac{1}{2}\right)},
$$

then we have $C^{\prime} \eta_{\nu, \alpha}^{*}=C^{\prime \prime} \eta_{\nu, \alpha}^{*}=\frac{1}{2}\left(\nu^{2}-1\right) \eta_{\nu, \alpha}^{*}$.

Proof. Since $\eta_{\nu, \alpha}^{*}(X)$ is a homogeneous function of degree $-(\nu+1)$, $L \eta_{\nu, \alpha}^{*}=-(\nu+1) \eta_{\nu, \alpha}^{*}$. On the other hand, $\Delta_{Q} \eta_{\nu, \alpha}^{*}=0$ as well as $\Delta_{Q} \eta_{\nu, \alpha}=0$. These combined with the formula (7) complete the proof. 
The function $\eta_{\nu, \alpha}^{*}(X)$ is also called a spherical harmonics of degree $-(\nu+1)$, which coincides with $\eta_{\nu, \alpha}(X)$ on the surface: $P(X)=1$.

\section{§2. Poincaré series and theta series}

2.1. Let $\mathfrak{o}$ be the ring of integers of an imaginary quadratic field of odd prime discriminant $-D$. It is, as in the introduction, assumed that the class number is one and the units are 1 and -1 only. Let us define a lattice $\mathfrak{L}$ of $V$ over $Z$ by $\mathfrak{L}=V \cap M_{2}(\mathfrak{o})$, and denote by $\mathfrak{L}(\ell)$ the subset of $\mathbb{Q}$ consisting of all elements of determinant $-\ell$ for each $\ell \in Z$. Let $\Lambda$ denote the discrete subgroup $S L_{2}$ (o) of $G$. Each subset $\mathfrak{L}(\ell)$ is $\Lambda$-invariant. For each positive integer $\ell$ we define a $C^{2 v+1}$-valued function on $G$ by

$$
h_{(\nu), \ell}(g)=\sum_{X \in \mathcal{R}(\ell)} \eta_{(\nu)}^{*}\left(X^{g}\right), \quad(g \in G),
$$

where $\eta_{(\nu)}^{*}(X)=\left(\eta_{\nu,-\nu}^{*}(X), \cdots, \eta_{\nu, \nu}^{*}(X)\right)$ and $\eta_{\nu, \alpha}^{*}(X)$ is given by (8). This series is, as we shall see below, absolutely convergent for $\nu>1$, hence the function $h_{(\nu), \ell}$ can be regarded as a type of Poincaré series, considering that the set $\mathcal{L}(\ell)$ is a union of some $\Lambda$-orbits. This combined with Lemmas 2 and 5 leads to

LEMMA 6. For each positive integer $\ell$ and $\nu>1$, it holds

(i) $h_{(\nu), \ell}(\gamma g \kappa)=h_{(\nu), \ell}(g) \rho_{2 \nu}(\kappa)$ for $\gamma \in \Lambda$ and $\kappa \in K$.

(ii) $C^{\prime} h_{(\nu), \ell}=C^{\prime \prime} h_{(\nu), \ell}=\frac{1}{2}\left(\nu^{2}-1\right) h_{(\nu), \ell}$.

For the purpose to prove that the series (9) is convergent absolutely and uniformly on any compact subset of $G$, it is sufficient to combine Lemma 4 with next two lemmas.

LEMMA 7. If $\ell$ is positive, a series $\sum_{X \in \mathfrak{R}(\ell)} P(X)^{-s}$ is absolutely convergent for $\operatorname{Re} s>1$.

Proof. By noting $P(X)=4 r \bar{r}+(m-n)^{2}$ for $X=\left(\begin{array}{cc}m & r \\ \bar{r} & n\end{array}\right) \in V$, divide the summation on $\mathfrak{L}(\ell)$ into the following three parts: one with $r=0$, one with $r \bar{r}=\ell$ and the others. The first, then, is a finite sum, and it can be easily seen that the second and the third are majorized by some constant multiples of $\sum_{n \in Z}\left(4 \ell+n^{2}\right)^{- \text {Res }}$ and $\sum_{r \in 0-\{0\}}(r \bar{r})^{-(\operatorname{Re} s-\varepsilon)}$ respectively, where $\varepsilon$ is chosen so that $\operatorname{Re} s-1>\varepsilon>0$. These imply the lemma. 
LEMMA 8. For arbitrary $g \in G$ there exists a positive constant $c$ such that $P\left(X^{g}\right) \geqq c P(X)$ for every $X \in V$ with non-positive determinant.

Proof. Since $P(X)$ is $K$-invariant, we can assume that $g$ is diagonal : $g=\left(\begin{array}{cc}\beta & 0 \\ 0 & \beta^{-1}\end{array}\right)$. Take $c$ to be the minimum of $|\beta|^{4}$ and $|\beta|^{-4}$, then we have $P\left(X^{g}\right)=4 r \bar{r}+\left(m|\beta|^{2}-n|\beta|^{-2}\right)^{2} \geqq P(X)+\left(m^{2}+n^{2}\right)(c-1)$, which is not less than $c P(X)$ because $P(X) \geqq m^{2}+n^{2}$ and $c \leqq 1$.

Remark. $h_{(\nu), \ell}$ vanishes for every odd $\nu$. Also $h_{(\nu), 0}$ can be defined by (9) in whose summation $X=0$ is excluded, and it is an Eisenstein series satisfying the properties in Lemma 6 .

2.2. For a function $f(z)$ on the upper half complex plane $\mathfrak{S}$ and a real matrix $\sigma=\left(\begin{array}{ll}a & b \\ c & d\end{array}\right)$ of positive determinant (i.e. $\sigma \in G L_{2}^{+}(R)$ ), we write

$$
\left(f \mid[\sigma]_{k}\right)(z)=(\operatorname{det}(\sigma))^{k / 2}(c z+d)^{-k} f(\sigma z)
$$

for $k \in Z$, where $\sigma z=(a z+b)(c z+d)^{-1}$. We define a differential operator $\delta_{\lambda}$ for $\lambda \in \boldsymbol{R}$ following Shimura ([12]) by

$$
\delta_{\lambda}=\frac{1}{2 \pi i}\left(\frac{\lambda}{2 i y}+\frac{\partial}{\partial z}\right)=\frac{1}{2 \pi i} y^{-\lambda} \frac{\partial}{\partial z} y^{\lambda},
$$

where $z=x+i y$, and we also put

$$
\delta_{\lambda}^{\ell}=\delta_{\lambda+2(\ell-1)} \cdots \delta_{\lambda+2} \delta_{\lambda} \quad \text { for } 0 \leqq \ell \in Z
$$

The "raising" operator $\delta_{\lambda}^{\ell}$ acts on functions on $\mathfrak{S}$ and satisfies that $\delta_{\lambda}^{\ell}\left(f \mid[\sigma]_{\lambda}\right)=\left(\delta_{\lambda}^{\ell} f\right) \mid[\sigma]_{\lambda+2 \ell}$ for every $\sigma \in G L_{2}^{+}(R)$. We need the following

LEMMA 9. (i) $\delta_{\lambda}^{\ell} \exp (2 \pi i a z)=\ell !(-4 \pi y)^{-\ell} L_{\ell}^{(\lambda-1)}(4 \pi a y) \exp (2 \pi i a z), \quad(\lambda$, $a \in \boldsymbol{R})$.

(ii) Put $t_{r}(z ; m, n)=\sqrt{y}(8 \pi y)^{-r / 2} H_{r}(\sqrt{2 \pi y}(m-n)) \exp (\pi i(x(-2 m n)$ $\left.\left.+i y\left(m^{2}+n^{2}\right)\right)\right)$, then $\delta_{r}^{\ell} t_{\gamma}=t_{\gamma+2 \ell},(0 \leqq \gamma \in Z, m, n \in \boldsymbol{R})$. Here $L_{\ell}^{(\lambda)}$ and $H_{\gamma}$ are Laguerre's and Hermite's polynomials respectively.

Proof. We can easily prove (i) or (ii) by induction on $\ell$, using a formula (cf. [8], p. 241, p. 252) :

$$
x \frac{d}{d x} L_{\ell}^{(\alpha)}(x)=(\ell+1) L_{\ell+1}^{(\alpha)}(x)-(\ell+\alpha+1-x) L_{\ell}^{(\alpha)}(x)
$$


or

$$
x \frac{d}{d x} H_{\ell}(x)=\left(x^{2}-(\ell+1)\right) H_{\ell}(x)-H_{\ell+2}(x),
$$

accordingly.

2.3. Now let us define a theta series with respect to the indefinite quadratic form $Q$ on $V$. Since the minimal majorant $R\left(X^{g}\right)$ is parametrized by $g \in G$, our function has double variables $z=x+i y \in \mathfrak{E}$ and $g \in G$. Namely we put

$$
\begin{aligned}
& \theta_{\nu, \alpha}(z, g)=\sqrt{y} \sum_{X \in \mathcal{I}} \eta_{\nu, \alpha}\left(X^{g}\right) \exp \left(\pi i\left(x Q(X)+i y R\left(X^{g}\right)\right)\right), \\
& \theta_{(\nu)}(z, g)=\left(\theta_{\nu,-\nu}(z, g), \cdots, \theta_{\nu, \nu}(z, g)\right),
\end{aligned}
$$

where $\mathfrak{L}=V \cap M_{2}(\mathfrak{D})$ as before. Obviously the series is absolutely convergent. We may, for non-triviality, assume that $\nu$ is even, since $\theta_{(\nu)}(z, g)$ vanishes for odd $\nu$. For abbreviation $\Gamma$ will stand for the subgroup $\Gamma_{0}(D)$ of $S L_{2}(Z)$ consisting of all $\sigma=\left(\begin{array}{ll}a & b \\ c & d\end{array}\right)$ with $D \mid c$, and $\chi$ denotes the character of $\Gamma$ defined by Kronecker's symbol, i.e. $\chi(\sigma)$ $=\left(\frac{-D}{d}\right)$ for $\sigma=\left(\begin{array}{ll}a & b \\ c & d\end{array}\right) \in \Gamma$.

LEMMA 10. For a non-negative even integer $\nu$, it holds that

(i) $\theta_{\nu, \alpha}(z, g)=\chi(\sigma) \theta_{\nu, \alpha}(z, g) \mid[\sigma]_{\nu+1}$ for every $\sigma \in \Gamma$.

(ii) $\theta_{(\nu)}(z, \gamma g \kappa)=\theta_{(\nu)}(z, g) \rho_{2 \nu}(\kappa)$ for every $\gamma \in \Lambda$ and $\kappa \in K$.

(ii.a) $\theta_{(\nu)}\left(z, g^{0}\right)=\theta_{(\nu)}(z, g) \rho_{2 \nu}\left({ }^{1}{ }_{-1}\right)$, where $g^{0}=\left({ }^{1}{ }_{-1}\right) g\left({ }^{1}{ }_{-1}\right)$.

(ii.b) $\theta_{(\nu)}(z, \bar{g})=\theta_{(\nu)}(z, g) \rho_{2 \nu}\left({ }_{1}^{-1}\right)$.

Proof. Due to Lemma 1.2 and Proposition 1.6 of Shintani [13], the theta transformation formula depends only on the form $Q$ and the lattice $\mathfrak{R}$, hence it is sufficient to prove (i) in the case that $\alpha=\nu$ and $g=1$ (the identity). Since $\eta_{\nu, \nu}(X)=r^{\nu}$ for $X=\left(\begin{array}{cc}m & r \\ \bar{r} & n\end{array}\right) \in \mathfrak{R}$, we can immediately see that $\theta_{\nu, \nu}(z, 1)=2 \theta_{-D}^{(\nu)}(z) \theta_{0}(z)$, where $\theta_{-D}^{(\nu)}(z)=\frac{1}{2} \sum_{r \in \in_{0}} r^{\nu}$ $\cdot \exp (2 \pi i r \bar{r} z)$ and $\theta_{0}(z)=\sqrt{y} \sum_{m, n \in Z} \exp \left(\pi i\left(x(-2 m n)+i y\left(m^{2}+n^{2}\right)\right)\right.$. As is well known, $\theta_{-D}^{(\nu)}=\chi(\sigma) \theta_{-D}^{(\nu)} \mid[\sigma]_{\nu+1}$ for $\sigma \in \Gamma$, and $\theta_{0}=\theta_{0} \mid[\sigma]_{0}$ for $\sigma \in S L_{2}(Z)$ since $\theta_{0}(z)=\sum_{c, d \in Z} \exp \left(-\pi y^{-1}|c z+d|^{2}\right)$ as derived by Poisson's summation formula. We have thus proved (i). For the proof of (ii) we have only to consider $\Lambda$-invariance of $\mathfrak{R}, K$-invariance of $R\left(X^{g}\right)$ and Lemma 2. We can derive (ii.a) in the same way as (ii), though $\left({ }^{1}{ }_{-1}\right)$ belongs 
to neither $A$ nor $K$. Finally from the definition (1) of $\eta_{\nu, \alpha}$ we have $\eta_{\nu, \alpha}(\bar{X})=(-1)^{\alpha} \eta_{\nu,-\alpha}(X)$, which imply (ii.b).

2.4. For a diagonal $g \in G$ the theta function $\theta_{\nu, \alpha}(z, g)$ splits into a convenient form. To describe this, we define two more theta series by

$$
\theta_{-D, \beta}^{(\alpha)}(z)=\frac{1}{2} \sum_{r \in \mathrm{o}} r^{\alpha} y^{-\beta} L_{\beta}^{(\alpha)}(4 \pi r \bar{r} y) \exp (2 \pi i r \bar{r} z)
$$

for $0 \leqq \alpha, \beta \in Z$, and

$$
\theta_{r}(z, v)=v^{r+1} y^{-r} \sum_{c, d \in Z}(c z+d)^{r} \exp \left(-\pi v^{2} y^{-1}|c z+d|^{2}\right)
$$

for $0 \leqq \gamma \in Z$, where $z=x+i y \in \mathfrak{F}$ and $0<v \in \boldsymbol{R}$. We abbreviate $\theta_{-D, 0}^{(\alpha)}$ to $\theta_{-D}^{(\alpha)}$ simply. We should notice that $\theta_{-D, \beta}^{(\alpha)}$ (or $\theta_{r}$ ) vanishes for odd $\alpha$ (or odd $\gamma$ ). Due to Lemma 9 we have $\theta_{-D, \beta}^{(\alpha)}=(-4 \pi)^{\beta}(\beta !)^{-1} \delta_{\alpha+1}^{\beta} \theta_{-D}^{(\alpha)}$ and $\theta_{r}=(-4)^{\gamma / 2} \delta_{0}^{\gamma / 2} \theta_{0}$ for even $\gamma$, so that $\theta_{-D, \beta}^{(\alpha)}=\chi(\sigma) \theta_{-D, \beta}^{(\alpha)} \mid[\sigma]_{\alpha+2 \beta+1}$ for $\sigma \in \Gamma$ and $\theta_{r}=\theta_{r} \mid[\sigma]_{r}$ for $\sigma \in S L_{2}(Z)$. There is another expression for $\theta_{r}$ :

$$
\theta_{r}(z, v)=(-4)^{r / 2} \sum_{m, n \in Z} t_{r}\left(z ; m v, n v^{-1}\right)
$$

where $t_{r}(z ; m, n)$ is the same as defined in Lemma 9. We can obtain (13) directly by Poisson's summation formula, or by applying the raising operator $\delta_{0}^{r / 2}$ to the simpler case of $\gamma=0$.

Now let us put $g(v)=\frac{1}{\sqrt{v}}\left({ }^{v}{ }_{1}\right)$ for $0<v \in R$. Then we have $\theta_{\nu, \alpha}(z, g(v))=(-1)^{\alpha} \theta_{\nu,-\alpha}(z, g(v))$ which, especially, vanishes for odd $\alpha$.

LEMMA 11. For a non-negative even integer $\alpha$,

$$
\theta_{\nu, \alpha}(z, g(v))=2(\nu !) \sum_{\beta, \gamma} \frac{1}{(\alpha+\beta) ! \cdot \gamma !} i^{r}(4 \pi)^{-\beta} \theta_{-D, \beta}^{(\alpha)}(z) \theta_{\gamma}(z, v),
$$

where the sum is taken over $\beta \geqq 0, \gamma \geqq 0$ with $2 \beta+\gamma=\nu-\alpha$.

Proof. Observing that

$$
x Q(X)+i y R\left(X^{g}\right)=(2 r \bar{r} z)+\left(x(-2 m n)+i y\left(m^{2} v^{2}+n^{2} v^{-2}\right)\right)
$$

for $g=g(v)$ and $X=\left(\begin{array}{cc}m & r \\ \bar{r} & n\end{array}\right) \in \mathfrak{R}$, and considering the explicit formula for $\eta_{\nu, \alpha}(X)$ in Lemma 3 , we can easily obtain the lemma. 


\section{§3. The Doi-Naganuma lifting}

3.1. We hereafter make the assumption that $\nu$ is even and positive. $\mathscr{S}_{\nu+1}$ will, as in the introduction, denote the space of all holomorphic cusp forms of weight $\nu+1$ of Neben type $\chi$ with respect to $\Gamma$, so that $f$ in $\mathscr{S}_{\nu+1}$ satisfies $f=\chi(\sigma) f \mid[\sigma]_{\nu+1}$ for $\sigma \in \Gamma$, where $\Gamma=\Gamma_{0}(D)$ and $\chi(\sigma)$ $=\left(\frac{-D}{d}\right)$ for $\sigma=\left(\begin{array}{ll}a & b \\ c & d\end{array}\right) \in \Gamma$. Let $f$ be a cusp form in $\mathscr{S}_{\nu+1}$, and let us consider the following integral of Petersson's inner product type in view of (i) in Lemma 10:

$$
F_{\alpha}(g)=2^{\nu-2} i^{-(\nu+1)} D^{\nu / 2} \int_{\Gamma \backslash \mathfrak{\Phi}} \theta_{\nu, \alpha}(z, g) \overline{f_{T}(z)} y^{\nu-1} d x d y,
$$

and define a $C^{2 v+1}$-valued function $F(g)$ on $G$ by

$$
F(g)=\left(F_{-\nu}(g), \cdots, F_{\nu}(g)\right),
$$

where $f_{T}$ in $\mathscr{S}_{\nu+1}$ is defined by $f_{T}(z)=\left(\overline{\left.f \mid[W]_{\nu+1}\right)(-\bar{z}}\right)$ with $W=\left({ }_{-D}{ }^{1}\right)$. In an obvious manner we can see that $\theta_{\nu, \alpha}(z, g)=O(\exp (-\varepsilon y))$ when $y \rightarrow \infty$ for some positive $\varepsilon$ and similar estimations hold at any other cusps, hence the integral (14) is absolutely convergent. The correspondence of $f$ with $F$ defines a linear map, which we shall denote by $I$, from $\mathscr{S}_{\nu+1}$ to a space of some functions on $G$. This is the definition of the Doi-Naganuma lifting in our case.

THEOREM 1. The lifted image $F=I(f)$ satisfies the followings:

(i) $F(\gamma g \kappa)=F(g) \rho_{2 \nu}(\kappa)$ for $\gamma \in \Lambda$ and $\kappa \in K$.

(i.a) $F\left(g^{0}\right)=F(g) \rho_{2 \nu}\left({ }^{1}{ }_{-1}\right)$, where $g^{0}=\left({ }^{1}{ }_{-1}\right) g\left({ }^{1}{ }_{-1}\right)$.

(i.b) $F(\bar{g})=F(g) \rho_{2 \nu}\left({ }_{1}^{-1}\right)$.

(ii) $C^{\prime} F=C^{\prime \prime} F=\frac{1}{2}\left(\nu^{2}-1\right) F$, where $C^{\prime}, C^{\prime \prime}$ are the Casimir operators on G.

Proof. (i), (i.a) and (i.b) are immediate consequences of (ii), (ii.a) and (ii.b) in Lemma 10, respectively. To prove (ii), we should recall that the space $\mathscr{S}_{\nu+1}$ is generated by Poincaré series for $1 \leqq \ell \in Z$ :

$$
p_{\ell}(z)=\sum_{\sigma \in \Gamma_{\infty} \backslash \Gamma} \chi(\sigma) \exp (2 \pi i \ell z) \mid[\sigma]_{\nu+1},
$$

where $\sigma$ runs over $\Gamma$ modulo the stabilizer $\Gamma_{\infty}$ of $\infty$ in $\Gamma$. We can easily obtain 


$$
\int_{\Gamma \backslash \mathfrak{g}} \theta_{(\nu)}(z, g) \overline{p_{\ell}(z)} y^{\nu-1} d x d y=\pi^{-\left(\nu+\frac{1}{2}\right)} \Gamma\left(\nu+\frac{1}{2}\right) h_{(\nu), \ell}(g),
$$

where $h_{(\nu), \ell}(g)$ is the same as defined by (9). The computation is valid for $\nu>1$. This combined with Lemma 6 implies (ii).

3.2. To give the Fourier expansion of the lifted image $F=I(f)$ we need some notations as follows. We should first recall that $\Lambda$ has the unique non-equivalent cusp, say $\infty$. Let us put $g(u, v)=\frac{1}{\sqrt{v}}\left(\begin{array}{ll}v & u \\ & 1\end{array}\right)$ for $u \in C$ and $0<v \in R$, so that every element of $G$ has unique expression as $g(u, v) \kappa$ with some $g(u, v)$ and $\kappa \in K$. We may abbreviate $g(0, v)$ to $g(v)$ as before. We put $S(u)=u+\bar{u}$ for $u \in C . K_{\alpha}(v)$ denotes the modified Bessel function of order $\alpha$ (e.g. [8], Chap. III). For each $\alpha \in Z$ a grössen character $\xi^{\alpha}$ is defined by $\xi^{\alpha}(r)=r^{\alpha}|r|^{-\alpha}$ for $r \in \mathfrak{D}-\{0\}$. Finally we put $\omega=\frac{1}{\sqrt{-D}}$, so that $(\omega)$ is the complementary ideal of $\mathfrak{o}$.

THEOREM 2. Let $f \in \mathscr{S}_{\nu+1}$ and $F=\left(F_{-\nu}, \cdots, F_{\nu}\right)$ be the lifted image $I(f)$. Suppose we have

$$
f(z)=\sum_{n=1}^{\infty} a(n) \exp (2 \pi i n z), \quad f_{T}(z)=\sum_{n=1}^{\infty} \overline{b(n)} \exp (2 \pi i n z),
$$

and put

$$
\begin{gathered}
C(0)=2^{\nu-1} D^{\nu / 2} \int_{\Gamma \backslash \mathfrak{\Phi}} f(z) \overline{\theta_{-D}^{(\nu)}(z)} y^{\nu-1} d x d y, \\
C(r)=C_{1}(r)+C_{2}(r) \quad \text { for } r \in \mathfrak{D}-\{0\} ; \\
C_{1}(r)=\sum_{n \backslash(r)} n^{\nu} a\left(n^{-2} r \bar{r}\right), \quad C_{2}(r)=(-i) D^{\nu / 2} \sum_{n \backslash(r \omega)} n^{\nu} b\left(n^{-2} D^{-1} r \bar{r}\right) .
\end{gathered}
$$

Then

$$
F_{\alpha}(g(u, v))=\sum_{r \in 0} C(r) \phi_{\alpha}(v, r) \exp (2 \pi i S(\bar{r} \omega u))
$$

for $|\alpha| \leqq \nu$, where

$$
\phi_{\alpha}(v, 0)=\delta_{\nu,|\alpha|} v, \quad \phi_{\alpha}(v, r)=\left(\begin{array}{c}
2 \nu \\
\nu-\alpha
\end{array}\right) \xi^{\alpha}(r) K_{\alpha}(4 \pi|r \omega| v) v^{\nu+1} \quad \text { for } r \neq 0
$$

We shall prove Theorem 2 in 3.5 after some preliminary lemmas. On the other hand, Theorem 2 says that the image $F=I(f)$ is not always cuspidal even though $f$ is a cusp form. To make clear we state 
this as follows, while the proof is obvious:

THEOREM 3. Let $\mathscr{S}_{\nu+1}^{1}$ be the orthogonal complement of $\theta_{-D}^{(\nu)}$ with respect to the Petersson metric in $\mathscr{S}_{\nu+1}$, then the lifted image $F=I(f)$ of $f$ in $\mathscr{S}_{v+1}^{1}$ satisfies the followings:

(iii) $F(g)^{t} \overline{F(g)}$ is bounded on $G$.

(iv) $\int_{C / 0} F(g(u, 1) \cdot g) d u d \bar{u}=0$ for every $g \in G$.

3.3. The next and following two lemmas are preparations to prove Theorem 2.

LEMMA 12. Under the same conditions and notations as in Theorem 2 ,

$$
F_{\alpha}(g(v))=\sum_{r \in \mathrm{o}} C(r) \phi_{\alpha}(v, r)
$$

Proof. We first notice that $F_{\alpha}(g(v))$ vanishes for odd $\alpha$ as well as $\theta_{\nu, \alpha}(z, g(v))$, and $F_{\alpha}(g(v))=F_{-\alpha}(g(v))$ for even $\alpha$. Hence we may assume that $\alpha$ is even and non-negative. We prove (17) by a direct computation of the integral (14) for $g=g(v)$. Owing to Lemma 11, it is reduced to a computation of an integral

$$
\int_{\Gamma \backslash \mathfrak{S}} \theta_{-D, \beta}^{(\alpha)}(z) \theta_{\gamma}(z, v) \overline{f_{T}(z)} y^{\nu-1} d x d y
$$

with $2 \beta+\gamma=\nu-\alpha, \beta \geqq 0, \gamma \geqq 0$. By the definition (12) of $\theta_{\gamma}$, it is plain to see that

$$
\theta_{\gamma}(z, v)=\delta_{\gamma, 0} v+\theta_{\gamma}^{1}(z, v)+D^{r / 2} \theta_{\gamma}^{1}(D z, v) \mid[W]_{r},
$$

where $\theta_{r}^{1}(z, v)=2 \sum_{n=1}^{\infty} n^{r} \sum_{\sigma \in \Gamma_{\infty} \backslash \Gamma} k(z, n) \mid[\sigma]_{\gamma}, k(z, n)=v^{\gamma+1} y^{-r} \exp \left(-\pi v^{2} n^{2} y^{-1}\right)$ and $W=\left({ }_{-}{ }^{1}\right)$. Hence the integral (18) decomposes into three parts. For the first, because of Lemma 6 of [12] we have, when $\gamma=0$,

$$
\int_{\Gamma \backslash \mathfrak{Ð}} \theta_{-D, \beta}^{(\alpha)}(z) \overline{f_{T}(z)} y^{\nu-1} d x d y=0 \quad \text { unless } \beta=0(\alpha=\nu) .
$$

By usual method the second part is computed as follows:

$$
\begin{aligned}
& \int_{\Gamma \backslash \mathfrak{F}} \theta_{-D, \beta}^{(\alpha)}(z) \overline{f_{T}(z)} \theta_{r}^{1}(z, v) y^{\nu-1} d x d y \\
& =2 \sum_{n=1}^{\infty} n^{\gamma} \int_{\Gamma \backslash \mathscr{\Phi}} \theta_{-D, \beta}^{(\alpha)}(z) \overline{f_{T}(z)} k(z, n) y^{\nu-1} d x d y
\end{aligned}
$$




$$
=2 v^{\nu+1} \sum_{n=1}^{\infty} n^{r} \int_{0}^{\infty} d y y^{\nu-\gamma-1} \exp \left(-\pi v^{2} n^{2} y^{-1}\right) \int_{0}^{1} \theta_{-D, \beta}^{(\alpha)}(z) \overline{f_{T}(z)} d x
$$

Here we need a formula (cf. [5], p. 175 (33), though there is a misprint.) :

$$
\int_{0}^{\infty} \exp \left(-a t-\frac{b}{t}\right) L_{\beta}^{(\alpha)}(a t) t^{\alpha+\beta-1} d t=(-1)^{\beta} \frac{2}{\beta !} a^{-\alpha / 2} b^{\alpha / 2+\beta} K_{\alpha}(2 \sqrt{a b})
$$

for $a, b>0$. We thus obtain

$$
\begin{aligned}
& \int_{\Gamma \backslash \mathfrak{\Phi}} \theta_{-D, \beta}^{(\alpha)}(z) \overline{f_{T}(z)} \theta_{r}^{1}(z, v) y^{\nu-1} d x d y \\
& \quad=(-\pi)^{\beta}(\beta !)^{-1} 2^{1-\alpha} i^{\alpha+1} D^{-\nu / 2} \sum_{r \in \mathbb{0}-\{0\}} \xi^{\alpha}(r) C_{2}(r) K_{\alpha}(4 \pi|r \omega| v) v^{\nu+1}
\end{aligned}
$$

The same computation holds for the third part. Thus by using a relation $\nu ! \sum_{2 \beta+\gamma=\nu-\alpha} 2^{r}(\beta ! \gamma !(\alpha+\beta) !)^{-1}=\left(\begin{array}{c}2 \nu \\ \nu-\alpha\end{array}\right)$, we can complete the proof of (17).

3.4. LEMma 13. Suppose that $F=\left(F_{-\nu}, \cdots, F_{\nu}\right)$ is a function on $G$, satisfying the properties (i), (i.a), (i.b) and (ii) in Theorem 1, and an additional condition that $F_{\alpha}(g(v))=\delta_{\nu,|\alpha|} B(0) v+O(\exp (-\varepsilon v))$ for $v \rightarrow \infty$ with some constants $\varepsilon>0$ and $B(0)$. Then, $F_{\alpha}$ has a Fourier expansion as follows:

$$
F_{\alpha}(g(u, v))=\sum_{r \in \mathrm{o}} B(r) \phi_{\alpha}(v, r) \exp (2 \pi i S(\bar{r} \omega u)),
$$

where $\phi_{\alpha}(v, r)$ is the same as in Theorem 2 and the coefficient $B(r)$ $(=B(-r)=B(\bar{r}))$ does not depend on $\alpha$.

Proof. This lemma is due to Weil [14], Chap. VIII. In fact, put formally $F_{\alpha}(g(u, v))=\sum_{r \in_{0}} \psi_{\alpha}(v, r) \exp (2 \pi i S(\bar{r} \omega u))$, then each term satisfies the Beltrami operator's equation $\left(E_{0}\right)$ in [14], p. 72 for $r=0$ or $(E)$ in p. 74 for $r \neq 0$. So we have $\psi_{\alpha}(v, 0)=\delta_{\nu, \alpha} B(0) v$. For $r \neq 0$ it first follows that we can put $\psi_{\nu}(v, r)=B(r) \phi_{\nu}(v, r)$, and then we obtain $\psi_{\alpha}(v, r)=B(r) \phi_{\alpha}(v, r)$ recursively by using a formula $x K_{\alpha}^{\prime}(x)+\alpha K_{\alpha}(x)$ $=-x K_{\alpha-1}(x)$ (cf. [8], p. 67) and by noting a special role of the factor $\left(\begin{array}{c}2 \nu \\ \nu-\alpha\end{array}\right) \xi^{\alpha}(r)$. It follows from (i.a) and (i.b) that $B(r)=B(-r)=B(\bar{r})$.

LEMMA 14. For a non-negative integer $\ell$, it holds that 


$$
\left[\frac{\partial^{\ell}}{\partial \bar{u}^{\ell}} \theta_{\nu, \nu}(z, g(u, v))\right]_{u=0}=2(-2 \pi i)^{\ell} v^{-\ell} y^{\ell} \theta_{-D}^{(\nu+\ell)}(z) \theta_{\ell}(-z, v)
$$

Proof. We first note that $\eta_{\nu, \nu}\left(X^{g}\right)=(m u+r)^{\nu}$ does not depend on $\bar{u}$ for $X=\left(\begin{array}{cc}m & r \\ \bar{r} & n\end{array}\right) \in \mathfrak{R}$ and $g=g(u, v)$. Put $Y=\pi y R\left(X^{g}\right)$ which is a quadratic polynomial of $\bar{u}, Y_{1}=\frac{\partial}{\partial \bar{u}} Y$ and $Y_{2}=\frac{\partial^{2}}{\partial \bar{u}^{2}} Y$, so that it holds $(-1)^{\ell} \exp (Y) \frac{\partial^{\ell}}{\partial \bar{u}^{\ell}} \exp (-Y)=Y_{2}^{\ell / 2} H_{\ell}\left(Y_{1} Y_{2}^{-1 / 2}\right)$ with Hermite's polynomial $H_{\ell}$. Hence

$$
\begin{aligned}
{\left[\frac{\partial^{\ell}}{\partial \bar{u}^{\ell}} \exp (-Y)\right]_{u=0}=} & \left(-r v^{-1}\right)^{\ell}(2 \pi y)^{\ell / 2} H_{\ell}\left(\sqrt{2 \pi y}\left(m v+n v^{-1}\right)\right) \\
& \exp \left(-\pi y\left(2 r \bar{r}+m^{2} v^{2}+n^{2} v^{-2}\right)\right),
\end{aligned}
$$

which leads to the proof of (20).

3.5. Proof of Theorem 2. Since our function $F=I(f)$ satisfies the assumption of Lemma 13, we may write $F_{\alpha}(g(u, v))$ in the form of (19). A simple observation of this and (17) follows that $B(0)=C(0)$. Next we must prove that $B(r)=C(r)$ for any $r \in \mathfrak{o}-\{0\}$. Observing that $\xi^{\alpha}(\mathfrak{a}), B(\mathfrak{a})$ and $C(\mathfrak{a})$ are well defined for each ideal $\mathfrak{a}=(r)$ if $\alpha$ is even, it is sufficient for our purpose to show

$$
\sum_{\mathfrak{a}} \xi^{\alpha}(\mathfrak{a}) B(\mathfrak{a}) N \mathfrak{a}^{-s}=\sum_{\mathfrak{a}} \xi^{\alpha}(\mathfrak{a}) C(\mathfrak{a}) N \mathfrak{a}^{-s}
$$

for all even $\alpha \in \boldsymbol{Z}$ and $s \in \boldsymbol{C}$ with sufficiently large real part, where $\mathfrak{a}$ runs over all non-zero integral ideals of $\circ$. For $|\alpha| \leqq \nu$ we can get (21) by the Mellin transform

$$
\int_{0}^{\infty}\left(F_{\alpha}(g(v))-\delta_{\nu,|\alpha|} C(0) v\right) v^{2 s-(\nu+2)} d v,
$$

which, in fact, is equal to

$$
\frac{1}{2}\left(\begin{array}{c}
2 \nu \\
\nu-\alpha
\end{array}\right) D^{s}(2 \pi)^{-2 s} \Gamma\left(s+\frac{\alpha}{2}\right) \Gamma\left(s-\frac{\alpha}{2}\right) \sum_{a} \xi^{\alpha}(\mathfrak{a}) C(\mathfrak{a}) N a^{-s}
$$

if we use (17), or the same in which $C(\mathfrak{a})$ is replaced by $B(\mathfrak{a})$ if we use (19). For $|\alpha|>\nu$ we may assume $\alpha=\nu+\ell$ with positive even $\ell$ because $\xi^{-\alpha}(\mathfrak{a})=\xi^{\alpha}(\overline{\mathfrak{a}}), B(\mathfrak{a})=B(\overline{\mathfrak{a}})$ and $C(\mathfrak{a})=C(\overline{\mathfrak{a}})$. On one hand, it follows from (19) that 


$$
\left[\frac{\partial^{\ell}}{\partial \bar{u}^{\ell}} F_{\nu}(g(u, v))\right]_{u=0}=(-2 \pi)^{\ell} D^{-\ell / 2} \sum_{r \in \mathcal{0}-\{0\}} r^{\ell} B(r) \phi_{\nu}(v, r)
$$

On the other hand, this is equal to

$$
\int_{\Gamma \backslash \mathfrak{\oplus}}\left[\frac{\partial^{\ell}}{\partial \bar{u}^{\ell}} \theta_{\nu, \nu}(z, g(u, v))\right]_{u=0} \cdot \overline{f_{T}(z)} y^{\nu-1} d x d y
$$

which becomes $(-2 \pi)^{\ell} D^{-\ell / 2} \sum_{r \in 0-\{0\}} r^{\ell} C(r) \phi_{\nu}(v, r)$ through a similar computation to Lemma 12 by using Lemma 14. We can therefore complete the proof of (21) for $\alpha=\nu+\ell$ by Mellin transform again.

3.6. We give here some supplementary remarks on Dirichlet series and their Euler products. Let $F=I(f)$ be the lifted image of a cusp form $f$ in $\mathscr{S}_{v+1}$, so that $F$ has the Fourier expansion as in Theorem 2. Let us put

$$
\Phi_{\alpha}(s)=D^{s}(2 \pi)^{-2 s} \Gamma\left(s+\frac{\alpha}{2}\right) \Gamma\left(s-\frac{\alpha}{2}\right) \sum_{a} \xi^{\alpha}(\mathfrak{a}) C(\mathfrak{a}) N \mathfrak{a}^{-s}
$$

for even $\alpha \in Z$ and $s \in C$ with sufficiently large real part. Because ${ }^{t} g^{-1}$ $=\left(\begin{array}{ll}1 & -1\end{array}\right) g\left(\begin{array}{ll}1 & 1\end{array}\right)$ for $g \in G, F\left({ }^{t} g^{-1}\right)=F(g) \rho_{2 \nu}\left(\begin{array}{ll}-1 & 1\end{array}\right)=F(\bar{g})$ and so $F(g(v))=F\left(g\left(v^{-1}\right)\right)$. Hence a variant expression of the Mellin transform

$$
\begin{gathered}
\int_{1}^{\infty}\left(F_{\alpha}(g(v))-\delta_{\nu,|\alpha|} C(0) v\right)\left(v^{2 s-(\nu+1)}+v^{(\nu+1)-2 s}\right) \frac{d v}{v} \\
-\delta_{\nu,|\alpha|} C(0)\left(\frac{1}{2 s-\nu}-\frac{1}{\nu+2-2 s}\right)
\end{gathered}
$$

gives the meromorphic continuation of $\Phi_{\alpha}(s)$ and the functional equation $\Phi_{\alpha}(s)=\Phi_{\alpha}(\nu+1-s)$ for $|\alpha| \leqq \nu$. In particular, $\Phi_{\alpha}(s)$ is entire for $|\alpha|$ $<\nu$, while $\Phi_{ \pm \nu}(s)$ is entire if and only if $f \in \mathscr{S}_{\nu+1}^{1}$. For $\alpha=\nu+\ell$ with positive even $\ell$ (It should be noticed $\Phi_{\alpha}=\Phi_{-\alpha}$ in our case.), by Rankin's method in the convolution of $f$ and $\theta_{-D}^{(\alpha)}$ it is also possible to get the meromorphic continuation of $\Phi_{\alpha}(s)$ and the functional equation $\Phi_{\alpha}(s)$ $=\Phi_{\alpha}(\nu+1-s)$, while we can say no more about the holomorphy except the fact $\left(s-\frac{\alpha}{2}\right)\left(s-\frac{\alpha}{2}+1\right) \cdots\left(s-\frac{\alpha}{2}+(\ell-1)\right) \Phi_{\alpha}(s)$ is entire.

When $f$ in $\mathscr{S}_{\nu+1}$ is a normalized primitive form (i.e. a common eigen-function of all Hecke operators with $a(1)=1$ ), it is well known 
that $f_{T}=-i D^{-\nu / 2} a(D) f$ and so $b(n)=i D^{-\nu / 2} \overline{a(D n)}$. Hence we can easily show

$$
\sum_{\mathfrak{a}} \xi^{\alpha}(\mathfrak{a}) C(\mathfrak{a}) N \mathfrak{a}^{-s}=\prod_{\mathfrak{p}}\left(1-\xi^{\alpha}(\mathfrak{p}) C(\mathfrak{p}) N \mathfrak{p}^{-s}+\xi^{2 \alpha}(\mathfrak{p}) N \mathfrak{p}^{\nu-2 s}\right)^{-1}
$$

for every even integer $\alpha$. It should be also remarked that $C(p)=a(p)$, $a\left(p^{2}\right)+p^{\nu}$ or $a(p)+\overline{a(p)}$ according as $\left(\frac{-D}{p}\right)=1,-1$ or 0 for each prime $\mathfrak{p} \mid p$. In particular, it holds

$$
\sum_{a} C(\mathfrak{a}) N \mathfrak{a}^{-s}=\sum_{n=1}^{\infty} a(n) n^{-s} \cdot \sum_{n=1}^{\infty} \overline{a(n)} n^{-s}
$$

\section{§4. A characterization of $\theta_{-D}^{(\nu)}(z)$}

4.1. As an application of the Doi-Naganuma lifting we give a proof of the following

THEOREM 4. Let $f$ be a normalized primitive form in $\mathscr{S}_{\nu+1}$, and assume that all the eigen-values for Hecke operators are real, then $f=\theta_{-D}^{(\nu)}$.

Remark. Our proof will be based on the fact that the lifted image $I(f)$ is cuspidal if and only if $f$ is orthogonal to $\theta_{-D}^{(\nu)}$, i.e. $f \in \mathscr{S}_{\nu+1}^{1}$. In contrast with this, the lifted image of a holomorphic cusp form is always a Hilbert modular cusp form in the real quadratic field case. By using this fact we may derive an analogous result as follows: There are no such primitive forms of Neben type $\left(\Gamma_{0}(\Delta),\left(\frac{\Delta}{*}\right)\right)$ as all the eigen-values for Hecke operators are real, where $\Delta$ is a discriminant of a real quadratic field. In fact, we have already treated the case that the class number is one and $\Delta$ is odd (cf. [2], especially Cor. to Prop. 5).

4.2. We quote a lemma on Rankin's convolution. For the proof and some other details we can refer to [2], p. 91 and [1], Th. 3, the latter of which, however, contains an obvious mistake in its statement.

Let $f_{j}(z)=\sum_{n=1}^{\infty} a_{j}(n) \exp (2 \pi i n z)(j=1$ or 2$)$ be a normalized primitive form in $\mathscr{S}_{v+1}$, so that the corresponding Dirichlet series $\phi_{j}(s)$ $=\sum_{n=1}^{\infty} a_{j}(n) n^{-s}$ has the Euler product as follows:

$$
\phi_{j}(s)=\prod_{p} \phi_{j, p}(s)
$$




$$
\phi_{j, p}(s)^{-1}= \begin{cases}\left(1-\xi_{j} V\right)\left(1-\eta_{j} V\right) & \text { if } p \neq D, \\ \left(1-a_{j}(p) V\right) & \text { if } p=D,\end{cases}
$$

where $V=p^{-s}$, and $\xi_{j}, \eta_{j}$ are two roots of the equation $x^{2}-a_{j}(p) x$ $+\left(\frac{-D}{p}\right) p^{\nu}=0$ for each rational prime $p$. The convolution of these is defined by

$$
\begin{aligned}
& \psi\left(s ; f_{1}, \bar{f}_{2}\right)=\prod_{p} \psi_{p}(s) ; \\
& \psi_{p}(s)^{-1}= \begin{cases}\left(1-\xi_{1} \bar{\xi}_{2} V\right)\left(1-\xi_{1} \bar{\eta}_{2} V\right)\left(1-\eta_{1} \bar{\xi}_{2} V\right)\left(1-\eta_{1} \bar{\eta}_{2} V\right) & \text { if } p \neq D, \\
\left(1-a_{1}(p) \overline{a_{2}(p)} V\right)\left(1-\overline{a_{1}(p)} a_{2}(p) V\right) & \text { if } p=D .\end{cases}
\end{aligned}
$$

LEMMA 15. $\psi\left(s ; f_{1}, \bar{f}_{2}\right)$ can be meromorphically continued to the whole complex s-plane and satisfies a functional equation. It is entire if $f_{1} \neq f_{2}$ and it has a simple pole at $s=\nu+1$ if $f_{1}=f_{2}$.

4.3. Proof of Theorem 4. Suppose that $f(z)=\sum_{n=1}^{\infty} a(n) \exp (2 \pi i n z)$ is the Fourier expansion and put $f_{\rho}(z)=\sum_{n=1}^{\infty} \overline{a(n)} \exp (2 \pi i n z)$. We have to prove that $f \neq f_{\rho}$ if $f$ is a normalized primitive form in $\mathscr{S}_{\nu+1}^{1}$. Owing to Lemma 15, it is sufficient to prove that $\psi\left(s ; f, \bar{f}_{\rho}\right)$ is entire. Let $F$ $=I(f)$ denote the lifted image whose Fourier expansion is, we may assume, given by (16). We consider a Dirichlet series associated with $F$ defined by

$$
H(s)=\zeta_{-D}(2 s-2 \nu) \sum_{a} C(\mathfrak{a})^{2} N a^{-s},
$$

where $\zeta_{-D}$ is the Dedekind zeta function of the imaginary quadratic field of discriminant $-D$. By comparing their Euler products, we can obtain

$$
H(s)=\psi(s ; f, \bar{f}) \psi\left(s ; f, \bar{f}_{\rho}\right) .
$$

This is the same as Proposition 6 in [2], though only the real quadratic field case is treated there, hence we omit the detail here. Therefore we have only to prove:

LEMMA 16. If $f \in \mathscr{S}_{\nu+1}^{1}$, then $H(s)$ can be meromorphically continued to the whole complex s-plane and is holomorphic except a simple pole at $s=\nu+1$.

Proof. Observing that $F(g) J^{-2} \overline{F(g)}$ is left $\Lambda$ - and right $K$-invariant where $J$ denote the same matrix as in the proof of Lemma 4, let us 
consider an integral

$$
\Omega(s)=\int_{\bigwedge G / K} F(g) J^{-2} t \overline{F(g)} E^{*}(g, s-\nu) d \dot{g},
$$

where the invariant volume $d \dot{g}$ is given by $v^{-3} d u d \bar{u} d v$ for $g=g(u, v) \kappa$ $(\kappa \in K)$. In the above we put $E^{*}(g, s)=D^{s}(2 \pi)^{-2 s} \Gamma(2 s) \zeta_{-D}(2 s) E(g, s)$ with $E(g, s)=\sum_{r \in \Lambda_{\infty} \backslash \Lambda} v(\gamma g)^{s}(\operatorname{Re} s>1)$, where $v(g)=v$ for $g=g(u, v)_{\kappa}$ and $\Lambda_{\infty}$ is the subgroup consisting of all $\gamma=\left(\begin{array}{ll}a & b \\ c & d\end{array}\right) \in \Lambda$ with $c=0$. As is well known, $E^{*}(g, s)$ can be meromorphically continued to the whole complex $s$-plane and is holomorphic except two simple poles at $s=0,1$. We first notice that $F(g)$ decreases rapidly when $v(g) \rightarrow \infty$ because $f \in \mathscr{S}_{\nu+1}^{1}$. By so-called Rankin's method we obtain, on one hand, that the integral $\Omega(s)$ is absolutely convergent for all $s \in C$ except two simple poles at $s=\nu, \nu+1$, and on the other hand, that $\Omega(s)$ is a constant multiple of $D^{2 s}(2 \pi)^{-4 s} \Gamma(s)^{2} \Gamma(s-\nu)^{2} H(s)$ when $\operatorname{Re} s$ is sufficiently large. In these computations we need a formula

$$
\int_{0}^{\infty} K_{\alpha}(v)^{2} v^{2 s-1} d v=2^{2 s-3} \Gamma(s+\alpha) \Gamma(s-\alpha) \Gamma(s)^{2} \Gamma(2 s)^{-1}
$$

for $\operatorname{Re} s>2|\alpha|$ (e.g. [5], p. 334 (45)), and an elementary identity

$$
\sum_{\alpha=-\nu}^{\nu}\left(\begin{array}{c}
2 \nu \\
\nu-\alpha
\end{array}\right) \Gamma(s+\alpha) \Gamma(s-\alpha) \Gamma(2 s)^{-1}=\Gamma(s-\nu)^{2} \Gamma(2 s-2 \nu)^{-1} .
$$

Thus we complete the proof of Lemma 16, and so, that of Theorem 4.

\section{REFERENCES}

[1] T. Asai, On the Fourier coefficients of automorphic forms at various cusps and some applications to Rankin's convolution, J. Math. Soc. Japan 28 (1976), 48-61.

[2] - On certain Dirichlet series associated with Hilbert modular forms and Rankin's method, Math. Ann. 226 (1977), 81-94.

[ 3 ] K. Doi and H. Naganuma, On the functional equation of certain Dirichlet series, Invent. Math. 9 (1969), 1-14.

[ 4 ] A. Erdélyi et al., Higher transcendental functions, Vol. 2, McGraw-Hill, 1953.

[ 5 ] — - Tables of integral transforms, Vol. 1, McGraw-Hill, 1954.

[ 6 ] H. Jacquet, Automorphic forms on $G L(2)$, II, Lec. Notes in Math. 278, Springer, 1972.

[ 7 ] S. Kudla, Theta-functions and Hilbert modular forms, to appear.

[8] W. Magnus et al., Formulas and theorems for the special functions of mathematical physics, 3rd ed., Springer, 1966.

[9] H. Naganuma, On the coincidence of two Dirichlet series associated with cusp forms of Hecke's "Neben"-type and Hilbert modular forms over a real quadratic 
field, J. Math. Soc. Japan 25 (1973), 547-555.

[10] S. Niwa, Modular forms of half integral weight and the integral of certain thetafunctions, Nagoya Math. J. 56 (1974), 147-161.

[11] T. Oda, On modular forms associated with indefinite quadratic forms of signature $(2, n-2)$, to appear.

[12] G. Shimura, The special values of zeta functions associated with cusp forms, Comm. Pure appl. Math. 29 (1976), 783-804.

[13] T. Shintani, On construction of holomorphic cusp forms of half integral weight, Nagoya Math. J. 58 (1975), 83-126.

[14] A. Weil, Dirichlet series and automorphic forms, Lec. Notes in Math. 189, Springer, 1971.

Department of Mathematics

Faculty of General Education

Ehime University 